PREPARED FOR THE U.S. DEPARTMENT OF ENERGY, UNDER CONTRACT DE-AC02-76CH03073

PPPL-3521

PPPL-3521

UC-70

Resistive Instabilities in Hall Current Plasma Discharge

by

Andrei A. Litvak and Nathaniel J. Fisch

November 2000
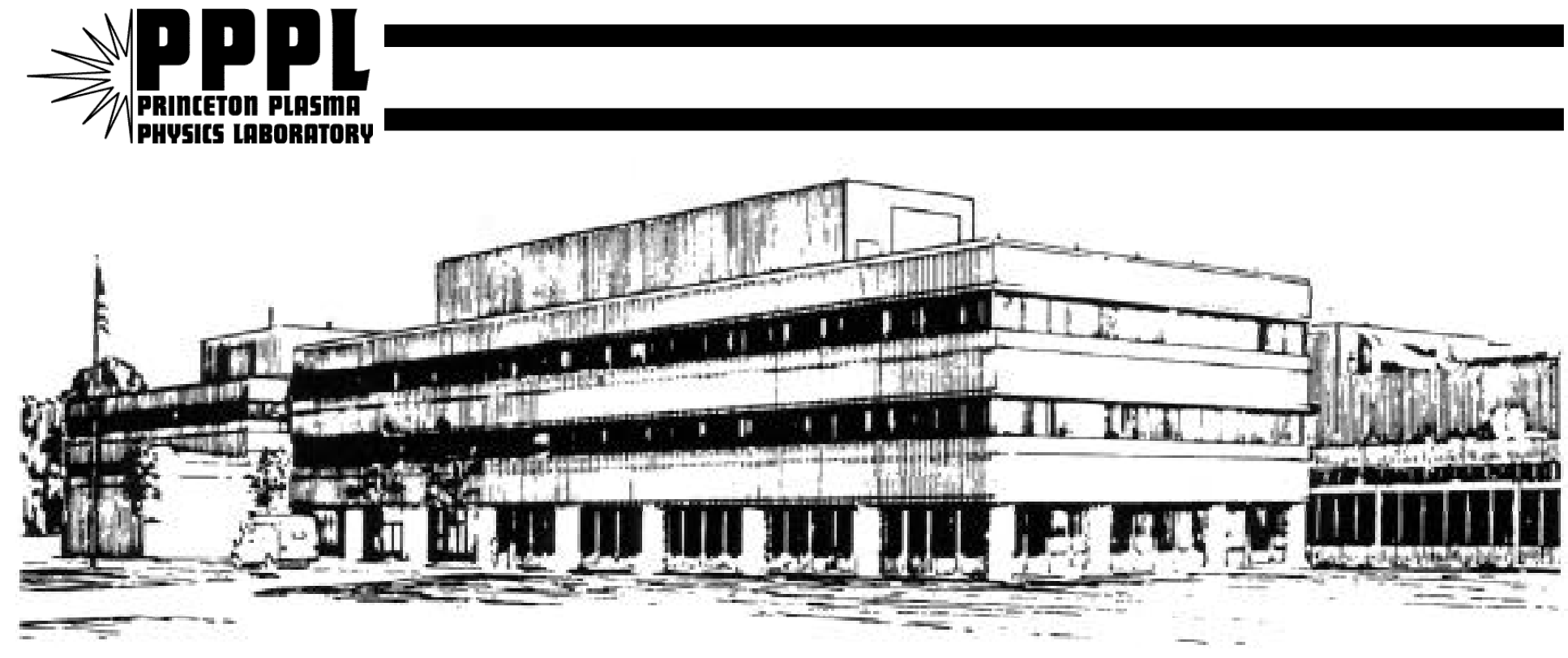

PRINCETON PLASMA PHYSICS LABORATORY PRINCETON UNIVERSITY, PRINCETON, NEW JERSEY 


\section{PPPL Reports Disclaimer}

This report was prepared as an account of work sponsored by an agency of the United States Government. Neither the United States Government nor any agency thereof, nor any of their employees, makes any warranty, express or implied, or assumes any legal liability or responsibility for the accuracy, completeness, or usefulness of any information, apparatus, product, or process disclosed, or represents that its use would not infringe privately owned rights. Reference herein to any specific commercial product, process, or service by trade name, trademark, manufacturer, or otherwise, does not necessarily constitute or imply its endorsement, recommendation, or favoring by the United States Government or any agency thereof. The views and opinions of authors expressed herein do not necessarily state or reflect those of the United States Government or any agency thereof.

\section{Availability}

This report is posted on the U.S. Department of Energy's Princeton Plasma Physics Laboratory Publications and Reports web site in Calendar Year 2000. The home page for PPPL Reports and Publications is: http://www.pppl.gov/pub_report/

DOE and DOE Contractors can obtain copies of this report from:

U.S. Department of Energy

Office of Scientific and Technical Information

DOE Technical Information Services (DTIS)

P.O. Box 62

Oak Ridge, TN 37831

Telephone: (865) 576-8401

Fax: (865) 576-5728

Email: reports@adonis.osti.gov

This report is available to the general public from:

National Technical Information Service

U.S. Department of Commerce

5285 Port Royal Road

Springfield, VA 22161

Telephone: $1-800-553-6847$ or

(703) $605-6000$

Fax: (703) 321-8547

Internet: http://www.ntis.gov/ordering.htm 


\title{
Resistive instabilities in Hall current plasma discharge
}

\author{
Andrei A. Litvak and Nathaniel J. Fisch \\ Princeton Plasma Physics Laboratory \\ PO Box 451, Princeton, NJ 08543
}

October 2, 2000

\begin{abstract}
Plasma perturbations in the acceleration channel of a Hall thruster are found to be unstable in the presence of collisions. Both electrostatic lower-hybrid waves and electromagnetic Alfven waves transverse to the applied electric and magnetic field are found to be unstable due to collisions in the $E \times B$ electron flow. These results are obtained assuming a two-fluid hydrodynamic model in slab geometry. The characterisitic frequencies of these modes are consistent with experimental observations in Hall current plasma thrusters.
\end{abstract}




\section{Introduction}

Hall thrusters are now being tested and seriously considered for propelling the next generation of spacecraft. One of the important issues for the successful operation of the Hall current plasma thrusters is the presence of plasma oscillations. This issue has been recognized since the earliest investigations (see, for example, Ref. [1]). Apart from their interactions with the power processing circuits, these oscillations play an important role in controlling the transport, conduction and mobility in Hall thrusters, directly affecting the thruster performance.

Careful consideration must also be given to possible interactions with the satellite electromagnetic systems used for communication and navigation. The possible influence of thruster plumes on communication signal propagation is of considerable interest [2]. Nonetheless, the electromagnetic interference, produced by the thruster during normal operation, is rarely studied and its driving mechanisms are not sufficiently understood.

For example, the model of hydrodynamic plasma instabilities, usually considered in the literature [3], despite its general agreement with the experimental data [4], does not explain all of the observations. Azimuthally propagating waves in the acceleration zone of the thruster are observed [5] even when the instability condition given in Ref.[3] is not satisfied.

In this paper we study two-dimensional plasma perturbations in a Hall current plasma thruster using two-fluid hydrodynamic theory. Instead of looking at the gradient-driven instabilities, we identify and consider the destabilizing effects associated with electron collisions. Despite the widely recognised importance of electron collisions for thruster operation, most of the models for plasma oscillations do not include collisional terms. Yet, we do find oscillations characteristic of Hall thrusters to be destabilized precisely by electron collisions.

We consider also electromagnetic waves. Perturbations of an electromagnetic nature, namely Alfven waves, were not considered in Ref.[3]. The characteristic plasma frequency and velocity scales found will then apply only for a small fraction of possible Hall thruter configurations and operation regimes. The limitations of the electrostatic-only approach were pointed out in Ref.[6]; however, the stability analysis of Alfven waves can not be found in the available literature.

The resistive instability discussed in this paper is in fact a special case of the more general process wherein an instability is driven by coupling to 
a dissipative process. Such effects have been well studied in the literature, such as plasma flow in the presence of a resistive-wall [7]. In the present case, although the driving mechanism for the instability is different from the resistive-wall instability [8], it is clear that the instability will occur only due to the interaction of the wave with the electron $E \times B$ flow in the presence of electron collisions.

This paper is organized as follows: In Sec. II, we write the model equations. In Sec. III, we derive the dispersion relation for unstable electrostatic modes, namely lower-hybrid waves. In Sec. III, the growth rate for unstable electromagnetic modes is found. Sec. IV offers a dicussion and summary of our results.

\section{Model Equations}

Let us consider Hall thruster two-component plasma, consisting of ions and electrons immersed in the magnetic field $B_{o}$, such that on the scale of the device electrons are magnetized, while ions are unmagnetized:

$$
\rho_{e}<<L<\rho_{i}
$$

In order to simlify the problem we neglect he variations of electric field and plasma density in the radial direction. We also neglect the axial component of the magnetic field. Such purely radial magnetic filed in the thruster channel will diverge with its magnitude being inversely proportional to the radius. Therefore the drift velocity of the electrons will be proportional to the radial position in the channel. However the angular velocity of the electrons around the channel will be constant. Therefore there will be no shear in the electron flow. Such characteristic of the the electron flow is ususally called the isodrift flow. Thus we can simplify the problem to purely two-dimesional by neglecting the channel curvaure and considering slab geometry. For simplicity in the following analysis we also neglect all plasma inhomogeneities, assuming variations of both density and magnetic field along the channel to be small.

The ion motion is governed by the following set of fluid equations

$$
\begin{aligned}
\frac{\partial N_{i}}{\partial t}+\nabla\left(\vec{v}_{i} N_{i}\right) & =0 \\
\frac{\partial \vec{v}_{i}}{\partial t}+\left(\vec{v}_{i} \cdot \nabla\right) \vec{v}_{i} & =\frac{e}{M} \vec{E}
\end{aligned}
$$


The zeroth order solution is the axial flow of unmagnetized ions being accelerated by the electric field in the channel according to

$$
v_{0} \frac{d v_{0}}{d x}=\frac{e}{M} E_{0}
$$

The electron motion is accordingly governed by the set of continuity and momentum equations:

$$
\begin{aligned}
& \frac{\partial N_{e}}{\partial t}+\nabla\left(\vec{v}_{e} N_{e}\right)=0 \\
& \frac{\partial \vec{v}_{e}}{\partial t}+\left(\vec{v}_{e} \cdot \nabla\right) \vec{v}_{e}=-\frac{e}{m}\left(\vec{E}+\frac{1}{c} \vec{v}_{e} \times \vec{B}\right)-\nu_{e} \vec{v}_{e}
\end{aligned}
$$

In the zeroth order, assuming $\nu_{e} \ll \Omega_{e}$ the electrons move in the $\hat{y}$ direction with the drift velocity,

$$
u_{0}=-c \frac{E_{0}}{B_{0}}
$$

The linearized system for the small perturbations of ion and electron densities and velocities can then be written as follows:

$$
\begin{gathered}
\frac{\partial n_{i}}{\partial t}+\overrightarrow{v_{0}} \frac{\partial n_{i}}{\partial x}+n_{0} \nabla \cdot \vec{v}_{i}=0 \\
\frac{\partial \vec{v}_{i}}{\partial t}+v_{0} \frac{\partial \vec{v}_{i}}{\partial x}=\frac{e}{M} \vec{E}_{1} \\
\frac{\partial n_{e}}{\partial t}+\overrightarrow{u_{0}} \frac{\partial n_{e}}{\partial y}+n_{0}\left(\nabla \cdot \vec{v}_{e}\right)=0 \\
\frac{\partial \vec{v}_{e}}{\partial t}+u_{0} \frac{\partial \vec{v}_{e}}{\partial y}=-\frac{e}{m}\left(\vec{E}_{1}+\frac{1}{c} \vec{v}_{e} \times \vec{B}_{0}+\frac{1}{c} \overrightarrow{u_{0}} \times \vec{B}\right)-\nu_{e} \vec{v}_{e}
\end{gathered}
$$

\section{Electrostatic Lower-Hybrid Waves}

Let us now consider electrostatic perturbations in the presence of electron collisions. Then the perturbation of electric filed is determined by the perturbation of the potential $\vec{E}_{1}=-\nabla \phi$. Considering oscillatory perturbations 
$A \propto A_{o} \exp (i \omega t-i \vec{k} \vec{r})$ The fluid equations for the two species will yield the following solutions for density perturbations

$$
\begin{aligned}
n_{i} & =\frac{k^{2} e N_{i 0}}{M\left(\omega-k_{x} v_{0}\right)^{2}} \phi \\
n_{e} & =\frac{k^{2} e N_{e 0}}{m \Omega_{e}^{2}} \phi-i \frac{e N_{0} \nu_{e} k^{2}}{m\left(\omega-k_{y} u_{0}\right) \Omega_{e}^{2}} \phi
\end{aligned}
$$

We have introduced here gyrofrequencies and plasma frequencies for ions and electrons $\Omega_{i, e}=e B_{o} / m_{i, e} c$ and $\omega_{i, e}^{2}=4 \pi e^{2} N_{e o} / m_{i, e}$ respectively. Also we assume

$$
\Omega_{i} \ll \omega \ll \Omega_{e}
$$

which is mostly valid for the oscillations, observed in Hall thrusters, where ion and electron gyrofrequencies are typically of the order of $10^{4}$ and $10^{9} \mathrm{~Hz}$ respectively.

Now we can substitute the obtained density perturbations into Poisson's equation

$$
-k^{2} \phi=4 \pi e\left(n_{e}-n_{i}\right)
$$

which will yield the following dispersion relation:

$$
1-\frac{\omega_{i}^{2}}{\left(\omega-k_{x} v_{0}\right)^{2}}+\frac{\omega_{e}^{2}}{\Omega_{e}^{2}}-\frac{\omega_{e}^{2}}{\Omega_{e}^{2}} \frac{i \nu_{e}}{\left(\omega-k_{y} u_{0}\right)}=0
$$

This is the dispersion relation for the Lower-Hybrid waves, modified to include collisions of the rotating electrons.

Consider now waves propagating along the $\hat{y}$ direction, so that $k_{x}=0$, which, in real thruster geometry, corresponds to azimuthally propagating waves. Under the assumption

$$
\omega \ll\left|k_{y} u_{0}\right|,
$$

which will be discussed later, the solutions for the dispersion relation (16) can be obtained as follows:

$$
\omega^{2}=\frac{\omega_{i}}{1+\frac{\omega_{e}^{2}}{\Omega_{e}^{2}}+\frac{i \nu_{e} \omega_{e}^{2}}{k_{y} u_{0} \Omega_{e}^{2}}}
$$

where the last term in the denominator in the RHS is small, therefore the final solution is

$$
\omega \approx \pm \omega_{L H}\left(1-\frac{i \nu_{e} \omega_{e}^{2}}{2 k_{y} u_{0}\left(\omega_{e}^{2}+\Omega_{e}^{2}\right)}\right)
$$


Here the lower-hybrid frequency is defined as

$$
\omega_{L H}^{2}=\frac{\omega_{i}^{2} \Omega_{e}^{2}}{\omega_{e}^{2}+\Omega_{e}^{2}}
$$

We have two modes, one of which will be unstable with the growth rate

$$
\gamma=\frac{\nu_{e}}{2 k_{y} u_{0}} \frac{\omega_{e}^{2}}{\left(\omega_{e}^{2}+\Omega_{e}^{2}\right)} \omega_{L H} \approx \pm \omega_{L H} \frac{\nu_{e}}{2 k_{y} u_{0}}
$$

Let us return to the assumption (17). In a typical Hall plasma thruster with the maximum applied magnetic filed $200 \mathrm{Gs}$, thruster channel diameter $10 \mathrm{~cm}$ and characteristic plasma density $10^{12} \mathrm{~cm}^{-3}$, the lower-hybrid frequency $\omega_{L H} \sim 10^{7} \mathrm{~Hz}$ can indeed be neglected compared to $k_{y} u_{0}$, which even for the smallest values of $k_{y}$ allowed by azimuthal symmetry will be at least order of magnitude larger. At the same time, a similar order of magnitude estimate for the increment of this instability, using effective electron collision frequency $\nu_{e} \sim 10^{6} \mathrm{~s}^{-1}$, yields $\gamma \sim 10^{6} \mathrm{~s}^{-1}$.

\section{Electromagnetic Waves}

For electromagnetic waves, it is no longer valid to represent the perturbations as functions of the electric potential only. The density and velocity perturbation can, however, be related to the electric field. The ion equations then remain almost unchanged:

$$
\begin{gathered}
v_{i x}=i \frac{1}{\left(\omega-k_{x} v_{0}\right)} \frac{e}{M} E_{x} \\
v_{i y}=i \frac{1}{\left(\omega-k_{x} v_{0}\right)} \frac{e}{M} E_{y} \\
n_{i}=\frac{e N_{0}}{M\left(\omega-k_{x} v_{0}\right)^{2}}\left(k_{x} E_{x}+k_{y} E_{y}\right)
\end{gathered}
$$

While for the electrons from the Eqns.(10,11), we obtain

$$
\begin{aligned}
v_{e x} & =\frac{1}{\Omega_{e}} \frac{e}{m} E_{y}-\frac{i\left(\omega-k_{y} u_{0}-i \nu_{e}\right)}{\Omega_{e}^{2}} \frac{e}{m} E_{x} \\
v_{e y} & =-\frac{1}{\Omega_{e}} \frac{e}{m} E_{x}-\frac{i\left(\omega-k_{y} u_{0}-i \nu_{e}\right)}{\Omega_{e}^{2}} \frac{e}{m} E_{y}+u_{0} \frac{B}{B_{0}}
\end{aligned}
$$




$$
\begin{array}{r}
n_{e}=\frac{i\left(\omega-k_{y} u_{0}-i \nu_{e}\right)}{\Omega_{e}^{2}\left(\omega-k_{y} u_{0}\right)} \frac{e N_{0}}{m}\left(k_{x} E_{x}+k_{y} E_{y}\right)+ \\
-\frac{e N_{0}}{m \Omega_{e} \omega}\left(k_{x} E_{y}-k_{y} E_{x}\right)
\end{array}
$$

We have used here the Maxwell's equation relating perturbations of electric and magnetic field, which in our case can be written as

$$
-i \omega \vec{B}=-c \hat{z}\left(k_{x} E_{y}-k_{y} E_{x}\right)
$$

The perturbation of the current $\vec{j}$ can be written as

$$
\begin{aligned}
& j_{x}=e\left[n_{i} v_{0}+N_{0}\left(v_{i x}-v_{e x}\right)\right] . \\
& j_{y}=e\left[-n_{e} u_{0}+N_{0}\left(v_{i y}-v_{e y}\right)\right] .
\end{aligned}
$$

The plasma dielectric tensor $\epsilon_{i k}$ can then be written as

$$
D_{i}=\epsilon_{i k} E_{k}=E_{k} \delta_{i k}+\frac{4 \pi}{i \omega} j_{i}\left(E_{k}\right) .
$$

The components of the dielectric tensor can then be found to be:

$$
\begin{aligned}
\epsilon_{x x} & =1+\frac{\left(\omega-k_{y} u_{0}\right) \omega_{e}^{2}}{\omega \Omega_{e}^{2}}-\frac{\omega_{i}^{2}}{\left(\omega-k_{x} v_{0}\right)^{2}} \\
\epsilon_{x y} & =-i \frac{\omega_{e}^{2}}{\omega \Omega_{e}}-\frac{\omega_{i}^{2} k_{y} v_{0}}{\omega\left(\omega-k_{x} v_{0}\right)^{2}} \\
\epsilon_{y y} & =1-\frac{\omega_{i}^{2}}{\omega\left(\omega-k_{x} v_{0}\right)}+\frac{\omega_{e}^{2}}{\Omega_{e}^{2}}+\frac{\omega_{e}^{2}}{\Omega_{e}^{2}} \frac{i \nu_{e}}{\left(\omega-k_{y} u_{0}\right)} \\
\epsilon_{y x} & =i \frac{\omega_{e}^{2}}{\Omega_{e} \omega}+\frac{\omega_{e}^{2} k_{x} u_{0}}{\omega \Omega_{e}^{2}}+\frac{\omega_{e}^{2} k_{x} u_{0}}{\omega \Omega_{e}^{2}} \frac{i \nu_{e}}{\left(\omega-k_{y} u_{0}\right)}
\end{aligned}
$$

The wave equation

$$
\left(k^{2} \delta_{i j}-k_{i} k_{j}-\frac{\omega^{2}}{c^{2}} \epsilon_{i j}\right) E_{j}=0
$$

yields the general notation for the dispersion relation, which can be written as

$$
k_{x}^{2} \epsilon_{x x}+k_{y} k_{x}\left(\epsilon_{x y}+\epsilon_{y x}\right)+k_{y}^{2} \epsilon_{y y}+\frac{\omega^{2}}{c^{2}}\left(\epsilon_{x x} \epsilon_{y y}-\epsilon_{x y} \epsilon_{y x}\right)=0
$$

If we once again consider only "azimuthal" propagation $k_{x}=0, k=k_{y}$, we have

$$
\frac{k^{2} c^{2}}{\omega^{2}}=\epsilon_{x x}-\frac{\epsilon_{x y} \epsilon_{y x}}{\epsilon_{y y}}
$$


We now substitute the notations for plasma dielectric tensor (32) - (35) to obtain the dispersion relation:

$$
\begin{aligned}
\frac{k^{2} v_{A}^{2}}{\omega^{2}} \frac{\omega_{i}^{2}}{\Omega_{i}^{2}} & =1-\frac{\omega_{i}^{2}}{\omega^{2}}+\frac{\omega_{i}^{2}}{\omega_{L H}^{2}} \frac{\left(\omega-k u_{0}-i \nu_{e}\right)}{\omega}- \\
& -\frac{\omega_{i}^{4}}{\Omega_{i}^{2}} \frac{\omega_{L H}^{2}}{\left(\omega^{2}-\omega_{i}^{2}\right) \omega_{L H}^{2}+\omega^{2} \omega_{i}^{2}\left(1-\frac{i \nu_{e}}{\omega-k u_{0}}\right)}
\end{aligned}
$$

Here we have introduced the Alfven velocity

$$
v_{A}^{2}=\frac{B_{0}^{2}}{4 \pi N_{0} M}
$$

and simplified (20) to

$$
\omega_{L H}^{2}=\frac{\omega_{i}^{2} \Omega_{e}^{2}}{\omega_{e}^{2}+\Omega_{e}^{2}} \simeq \Omega_{i} \Omega_{e}
$$

We seek the solution of (39) in the form of unstable wave with the frequency $\omega=\omega_{r}-i \gamma$, assuming $\gamma \ll \omega_{r}$. After some calculations using the estimate $\Omega_{i} \ll \omega, \omega_{i}, \omega_{L H}$, we obtain the solution as the Alfven wave

$$
\omega_{r} \approx k v_{A}
$$

which will be unstable with the growth rate

$$
\gamma=\frac{\nu_{e}}{2} \frac{v_{A}}{u_{0}} \frac{\omega_{r}^{2}}{\omega_{L H}^{2}} .
$$

For the typical value of Alfven velocity in Hall thrusters $v_{A} \sim 5 \cdot 10^{6} \mathrm{~cm} / \mathrm{s}$, the frequency of these waves will be $\omega_{r} \sim 10^{6} \mathrm{~Hz}$ and the growth rate $\gamma \sim 10^{4} \mathrm{~s}^{-1}$.

\section{Discussion and Conclusions}

We obtained linearly unstable solutions for azimuthally propagating waves in Hall thrusters. The purely azimuthal electrostatic lower-hybrid wave is shown to be driven unstable due to the resistive coupling to the electron drift flow, rather then, as commonly thought, driven unstable only by density and 
magnetic field gradients. The Alfven wave is also shown to be unstable due to the electron collisions.

For typical Hall thruster parameters, the electrostatic instability will occur for azimuthally propagating lower-hybrid waves with frequencies $\sim$ $10^{7} \mathrm{~Hz}$ and the electromagnetic instability for Alfven waves with frequencies $\sim 10^{6} \mathrm{~Hz}$. While the growth rate for Alfven waves are much smaller, on the timescales associated with the steady state thruster operation, both of these modes can become quite significant. It would be the nonlinear saturation mechanism, not considered here, rather than the linear growth rate, that would determine the relative importance of these mechanisms.

We also note, that for the obtained frequency ranges and modes the hydrodynamic description is quite appropriate. There is no collisionless damping or excitation in the direction transverse to the applied magnetic field, and for the direction parallel to the magnetic field kinetic effects could be neglected if $\omega \gg k_{z} v_{T e}$. This condiion is easily satisfied for the considered waves and thruster operation parameters.

Plasma oscillations in these frequency ranges were observed in multiple experimental studies of Hall thrusters [9]. It remains, however, to distinguish experimentally the resistive mechanism of instability of the modes considered here from the mechanism of instability due to field or density gradients. Observations of the instability in regions clearly gradient-free would support the theories advanced here.

\section{Acknowledgements}

The authors want to thank Dr. Y. Raitses for many fruitful discussions. The work was supported by U.S. DOE Contract No. DE-AC02-76-CHO-3073. 


\section{References}

[1] G.S.James, R.S.Lowder. "Anomalous Electron Diffusion and Ion Acceleration in a Low-Density Plasma." Physycs of Fluids, 9(6), 1115 (1966).

[2] S.G.Ohler, B.E.Gilchrist and A.D.Gallimore. "Electromagnetic Signal Modification in a Localized High-speed Plasma Flow: Simulations and Experimental Validation of a Stationary Plasma Thruster." IEEE Transactions on Plasma Science, 27(2), 587 (1999).

[3] Y,B.Esipchuk and G.N.Tilinin "Drift Instability in a Hall-curent plasma accelerator." Soviet Physics - Technical Physics, 21(4), 417 (1976).

[4] Y.B.Esipchuk, A.I.Morozov, G.N.Tilinin, A.V.Trofimov. "Plasma Oscillations in Closed Drift Accelerators with Extended Acceleration Zone." Soviet Physics - Technical Physics, 18(7), 928 (1974).

[5] Y.B.Esipchuk. "Studies of Plasma Systems with Closed Electron Drift and Distributed Electric Field." Plazmennye Uskoriteli, edited by L.A.Artsimovich. (Moscow, Mashinostroenie, 1973), p.75. (in Russian)

[6] E.Y.Choueiri. "Characterization of Oscillations in Closed Drift Thrusters." Proceedigs of the 30th Joint Propulsion Conference, Indianapolis, Indiana, 1994. AIAA 94-3013

[7] V.K.Neil, A.M.Sessler "Longitudinal Resistive Instabilitites of Intense Coasting Beams in Particla Accellerators." Review of Scientific Instruments, 36(4), 429 (1965)

[8] B.M.Veeresha, S.N.Bhattacharyya, K.Avinash. "Flow-driven Resistive Wall Instability." Physics of Plasmas, 6(12), 4479 (1999).

[9] V.V.Zhurin, H.R.Kaufman, and R.S.Robinson. "Physics of Closed Drift Thrusters." Plasma Sources Science Technology, 8, R1 (1999). 
The Princeton Plasma Physics Laboratory is operated by Princeton University under contract with the U.S. Department of Energy.

\author{
Information Services \\ Princeton Plasma Physics Laboratory \\ P.O. Box 451 \\ Princeton, NJ 08543
}

Phone: 609-243-2750

Fax: 609-243-2751

e-mail: pppl_info@pppl.gov

Internet Address: http://www.pppl.gov 